\title{
MADM Problems with Correlation Coefficient of Trapezoidal Fuzzy Intuitionistic Fuzzy Sets
}

\author{
John Robinson and Henry Amirtharaj \\ Bishop Heber College, Tiruchirappalli, Tamil Nadu 620017, India \\ Correspondence should be addressed to John Robinson; robijohnsharon@gmail.com
}

Received 4 May 2014; Accepted 8 July 2014; Published 4 August 2014

Academic Editor: Panagote M. Pardalos

Copyright ( 2014 J. Robinson and H. Amirtharaj. This is an open access article distributed under the Creative Commons Attribution License, which permits unrestricted use, distribution, and reproduction in any medium, provided the original work is properly cited.

\begin{abstract}
This paper discusses on the notion of trapezoidal fuzzy intuitionistic fuzzy sets (TzFIFSs) and some of the arithmetic operations of the same. Correlation coefficient of TzFIFS is proposed based on the membership, nonmembership, and hesitation degrees. The weighted averaging (WA) operator and the weighted geometric (WG) operator are proposed for TzFIFSs. Based on these operators and the correlation coefficient defined for the TzFIFS, new multiattribute decision making (MADM) models are proposed and numerical illustration is given.
\end{abstract}

\section{Introduction}

Intuitionistic fuzzy sets (IFSs) proposed by Atanassov [1-3] are a generalization of the concept of fuzzy sets. Atanassov and Gargov [4] expanded the IFSs, using interval value to express membership and nonmembership function of IFSs. Liu and Yuan [5] introduced the concept of fuzzy number IFSs as a further generalization of IFSs. Among the works done in IFSs, Szmidt and Kacprzyk [6-8] can be mentioned. To the best of our knowledge, Burillo et al. [9] proposed the definition of intuitionistic fuzzy number (IFN) and studied the perturbations of IFN and the first properties of the correlation between these numbers. Many researchers have applied the IFS theory to the field of decision making. Recently some researches $[5,10-14]$ showed great interest in the fuzzy number IFSs and applied it to the field of decision making. Based on the arithmetic aggregation operators, $\mathrm{Xu}$ and Yager [15], Xu and Chen [16, 17], and Wang [13] developed some new geometric aggregation operators and intuitionistic fuzzy ordered weighted averaging (IFOWA) operator. Szmidt and Kacprzyk [7] proposed some solution concepts in group decision making with intuitionistic (individual and social) fuzzy preference relations. Szmidt and Kacprzyk [8] investigated the consensus-reaching process in group decision making based on individual intuitionistic fuzzy preference relations. Herrera et al. [18] developed an aggregation process for combining numerical, interval valued, and linguistic information and then proposed different extensions of this process to deal with contexts in which information such as IFSs or multigranular linguistic information can appear. $\mathrm{Xu}$ and Yager [15] developed some geometric aggregation operators for MADM problems. Li [19] investigated MADM problems with intuitionistic fuzzy information and constructed several linear programming models to generate optimal weights for attributes.

Multiattribute decision making (MADM) problems are of importance in most kinds of fields such as engineering, economics, and management. It is obvious that much knowledge in the real world is fuzzy rather than precise. Imprecision comes from a variety of sources such as unquantifiable information [20]. In many situations decision makers have imprecise/vague information about alternatives with respect to attributes. It is well known that the conventional decision making analysis using different techniques and tools has been found to be inadequate to handle uncertainty of fuzzy data. To overcome this problem, the concept of fuzzy approach has been used in the evaluation of decision making systems. For a long period of time, efforts have been made in designing various decision making systems suitable for the arising dayto-day problems. MADM problems are widespread in reallife decision making situations and the problem is to find a 
desirable solution from a finite number of feasible alternatives assessed on multiple attributes, both quantitative and qualitative [21]. In order to choose a desirable solution, the decision maker often provides his/her preference information which takes the form of numerical values, such as exact values, interval number values, and fuzzy numbers. However, under many conditions, numerical values are inadequate or insufficient to model real-life decision problems. Indeed, human judgments including preference information may be stated in intuitionistic fuzzy information, especially in trapezoidal fuzzy intuitionistic fuzzy information. Hence, MADM problems under intuitionistic fuzzy or trapezoidal fuzzy intuitionistic fuzzy environment are an interesting area of study for researchers in the recent days.

It is well known that the conventional correlation analysis using probabilities and statistics has been found to be inadequate to handle uncertainty of failure data and modeling. The method to measure the correlation between two variables involving fuzziness is a challenge to classical statistical theory. Park et al. [22] proposed the correlation coefficient of interval valued intuitionistic fuzzy sets. Robinson and Amirtharaj [23-26] proposed correlation coefficient for vague sets, interval vague sets, triangular and trapezoidal IFSs, and a revised correlation coefficient for triangular and trapezoidal IFSs using graded mean integration representation. In this paper, a novel method of correlation coefficient of trapezoidal fuzzy intuitionistic fuzzy sets (TzFIFSs) is proposed and developed by taking into account the membership, nonmembership, and the hesitation degrees of TzFIFSs. The weighted averaging (WA) and weighted geometric (WG) operators for TzFIFSs are proposed for MADM problems. Based on these operators, an approach is suggested to solve uncertain multiple attribute group decision making problems, where the attribute values are trapezoidal fuzzy intuitionistic fuzzy numbers. A new algorithm is developed to solve the MADM problems in which the correlation coefficient of TzFIFSs is used for ranking alternatives.

\section{Arithmetic Operations for TzFIFS}

Arithmetic operations of TzFIFNs are based on the arithmetic operations of TzFNs. In TzFIFN the membership and nonmembership degrees take the form of trapezoidal fuzzy number. The basic concepts related to TzFIFNs are presented in the following.

Definition 1 (trapezoidal fuzzy number $(\mathrm{TzFN}))$. $A=$ $(a, b, c, d)$ is called a trapezoidal fuzzy number, if the membership function $\mu_{A}: R \rightarrow[0,1]$ is expressed as

$$
\mu_{A}(x)= \begin{cases}\frac{x-a}{b-a} & \text { for } a \leq x<b \\ \frac{1-x}{d-c} & \text { for } b \leq x<c \\ 0 & \text { for } c \leq x<d\end{cases}
$$

where $x \in R, 0 \leq a \leq b \leq c \leq d \leq 1$.
Definition 2 (trapezoidal fuzzy intuitionistic fuzzy number (TzFIFN)). Let $X$ be a nonempty set. Then $A=$ $\left\{\left\langle x, \mu_{A}(x), \gamma_{A}(x)\right\rangle / x \in X\right\}$ is called a trapezoidal fuzzy intuitionistic fuzzy number if $\mu_{A}(x)=\left(\mu_{A_{1}}(x), \mu_{A_{2}}(x)\right.$, $\left.\mu_{A_{3}}(x), \mu_{A_{4}}(x)\right)$ and $\gamma_{A}(x)=\left(\gamma_{A_{1}}(x), \gamma_{A_{2}}(x), \gamma_{A_{3}}(x), \gamma_{A_{4}}(x)\right)$ are trapezoidal fuzzy numbers, which can express the membership degree and the nonmembership degree of $x$ in $X$ and fulfill $0 \leq \mu_{A_{4}}(x)+\gamma_{A_{4}}(x) \leq 1$, for all $x \in X$.

An intuitionistic fuzzy number expressed on the basis of trapezoidal fuzzy number is called trapezoidal fuzzy intuitionistic fuzzy number.

$\pi_{A}(x)=\left(\pi_{A_{1}}(x), \pi_{A_{2}}(x), \pi_{A_{3}}(x), \pi_{A_{4}}(x)\right)$ is called the hesitation degree of the given trapezoidal fuzzy intuitionistic fuzzy set. Also we have $0 \leq \pi_{A_{1}}(x), \pi_{A_{2}}(x), \pi_{A_{3}}(x), \pi_{A_{4}}(x) \leq$ 1 , for all $x \in X$.

Suppose

$$
\begin{gathered}
A=\left\{\left\langlex,\left[\mu_{A_{1}}(x), \mu_{A_{2}}(x), \mu_{A_{3}}(x), \mu_{A_{4}}(x)\right],\right.\right. \\
\left.\left.\left[\gamma_{A_{1}}(x), \gamma_{A_{2}}(x), \gamma_{A_{3}}(x), \gamma_{A_{4}}(x)\right]\right\rangle \times(x \in X)^{-1}\right\}, \\
B=\left\{\left\langlex,\left[\mu_{B_{1}}(x), \mu_{B_{2}}(x), \mu_{B_{3}}(x), \mu_{B_{4}}(x)\right],\right.\right. \\
\left.\left.\left[\gamma_{B_{1}}(x), \gamma_{B_{2}}(x), \gamma_{B_{3}}(x), \gamma_{B_{4}}(x)\right]\right\rangle \times(x \in X)^{-1}\right\}
\end{gathered}
$$

are two TzFIFNs; then according to the above operation rules of intuitionistic fuzzy numbers and the operation rules of trapezoidal fuzzy numbers, the operational rules of TzFIFNs are as follows:

$$
\begin{gathered}
A+B=\left\{\left\langle\left[\mu_{A_{1}}(x), \mu_{A_{2}}(x), \mu_{A_{3}}(x), \mu_{A_{4}}(x)\right],\right.\right. \\
\left.\left[\gamma_{A_{1}}(x), \gamma_{A_{2}}(x), \gamma_{A_{3}}(x), \gamma_{A_{4}}(x)\right]\right\rangle \\
+\left\langle\left[\mu_{B_{1}}(x), \mu_{B_{2}}(x), \mu_{B_{4}}(x)\right],\right. \\
\left.\left.\left[\gamma_{B_{1}}(x), \gamma_{B_{2}}(x), \gamma_{B_{3}}(x), \gamma_{B_{4}}(x)\right]\right\rangle\right\} \\
=\left\{\left\langle\left[\mu_{A_{1}}(x)+\mu_{B_{1}}(x)-\mu_{A_{1}}(x) \cdot \mu_{B_{1}}(x),\right.\right.\right. \\
\mu_{A_{2}}(x)+\mu_{B_{2}}(x)-\mu_{A_{2}}(x) \cdot \mu_{B_{2}}(x), \\
\mu_{A_{3}}(x)+\mu_{B_{3}}(x)-\mu_{A_{3}}(x) \cdot \mu_{B_{3}}(x), \\
\left.\mu_{A_{4}}(x)+\mu_{B_{4}}(x)-\mu_{A_{4}}(x) \cdot \mu_{B_{4}}(x)\right], \\
{\left[\gamma_{A_{1}}(x) \cdot \gamma_{B_{1}}(x), \gamma_{A_{2}}(x) \cdot \gamma_{B_{2}}(x),\right.} \\
\left.\left.\left.\gamma_{A_{3}}(x) \cdot \gamma_{B_{3}}(x), \gamma_{A_{4}}(x) \cdot \gamma_{B_{4}}(x)\right]\right\rangle\right\},
\end{gathered}
$$




$$
\begin{aligned}
& A \cdot B=\left\{\left\langle\left[\mu_{A_{1}}(x), \mu_{A_{2}}(x), \mu_{A_{3}}(x), \mu_{A_{4}}(x)\right],\right.\right. \\
& \left.\left[\gamma_{A_{1}}(x), \gamma_{A_{2}}(x), \gamma_{A_{3}}(x), \gamma_{A_{4}}(x)\right]\right\rangle \\
& \cdot\left\langle\left[\mu_{B_{1}}(x), \mu_{B_{2}}(x), \mu_{B_{4}}(x)\right]\right. \text {, } \\
& \left.\left.\left[\gamma_{B_{1}}(x), \gamma_{B_{2}}(x), \gamma_{B_{3}}(x), \gamma_{B_{4}}(x)\right]\right\rangle\right\} \\
& =\left\{\left\langle\left[\mu_{A_{1}}(x) \cdot \mu_{B_{1}}(x), \mu_{A_{2}}(x) \cdot \mu_{B_{2}}(x)\right.\right.\right. \text {, } \\
& \left.\mu_{A_{3}}(x) \cdot \mu_{B_{3}}(x), \mu_{A_{4}}(x) \cdot \mu_{B_{4}}(x)\right], \\
& {\left[\gamma_{A_{1}}(x)+\gamma_{B_{1}}(x)-\gamma_{A_{1}}(x) \cdot \gamma_{B_{1}}(x),\right.} \\
& \gamma_{A_{2}}(x)+\gamma_{B_{2}}(x)-\gamma_{A_{2}}(x) \cdot \gamma_{B_{2}}(x), \\
& \gamma_{A_{3}}(x)+\gamma_{B_{3}}(x)-\gamma_{A_{3}}(x) \cdot \gamma_{B_{3}}(x), \\
& \left.\left.\left.\gamma_{A_{4}}(x)+\gamma_{B_{4}}(x)-\gamma_{A_{4}}(x) \cdot \gamma_{B_{4}}(x)\right]\right\rangle\right\}, \\
& \lambda A=\lambda\left(\left[\mu_{A_{1}}(x), \mu_{A_{2}}(x), \mu_{A_{3}}(x), \mu_{A_{4}}(x)\right]\right. \text {, } \\
& \left.\left[\gamma_{A_{1}}(x), \gamma_{A_{2}}(x), \gamma_{A_{3}}(x), \gamma_{A_{4}}(x)\right]\right) \\
& =\left\{\left\langle\left[1-\left(1-\mu_{A_{1}}(x)\right)^{\lambda}, 1-\left(1-\mu_{A_{2}}(x)\right)^{\lambda}\right.\right.\right. \text {, } \\
& \left.1-\left(1-\mu_{A_{3}}(x)\right)^{\lambda}, 1-\left(1-\mu_{A_{4}}(x)\right)^{\lambda}\right], \\
& {\left[\left(\gamma_{A_{1}}(x)\right)^{n},\left(\gamma_{A_{2}}(x)\right)^{n},\right.} \\
& \left.\left.\left.\left(\gamma_{A_{3}}(x)\right)^{n},\left(\gamma_{A_{4}}(x)\right)^{n}\right]\right\rangle\right\} \\
& A^{\lambda}=\left(\left[\mu_{A_{1}}(x), \mu_{A_{2}}(x), \mu_{A_{3}}(x), \mu_{A_{4}}(x)\right]\right. \text {, } \\
& \left.\left[\gamma_{A_{1}}(x), \gamma_{A_{2}}(x), \gamma_{A_{3}}(x), \gamma_{A_{4}}(x)\right]\right)^{\lambda} \\
& =\left\{\left\langle\left[\left(\mu_{A_{1}}(x)\right)^{n},\left(\mu_{A_{2}}(x)\right)^{n},\right.\right.\right. \\
& \left.\left(\mu_{A_{3}}(x)\right)^{n},\left(\mu_{A_{4}}(x)\right)^{n}\right], \\
& {\left[1-\left(1-\gamma_{A_{1}}(x)\right)^{\lambda}, 1-\left(1-\gamma_{A_{2}}(x)\right)^{\lambda}\right. \text {, }} \\
& \left.\left.\left.1-\left(1-\gamma_{A_{3}}(x)\right)^{\lambda}, 1-\left(1-\gamma_{A_{4}}(x)\right)^{\lambda}\right]\right\rangle\right\},
\end{aligned}
$$$$
\lambda \geq 0 \text {. }
$$

For the above operation rules, the following are true:

(i) $A+B=B+A$,

(ii) $A \cdot B=B \cdot A$,

(iii) $\lambda(A+B)=\lambda A+\lambda B, \lambda \geq 0$,

(iv) $\lambda_{1} A+\lambda_{2} A=\left(\lambda_{1}+\lambda_{2}\right) A, \lambda_{1}, \lambda_{2} \geq 0$,

(v) $A^{\lambda_{1}} \cdot A^{\lambda_{2}}=(A)^{\lambda_{1}+\lambda_{2}}, \lambda_{1}, \lambda_{2} \geq 0$.

\section{Correlation Coefficient of TzFIFS}

Let $X=\left\{x_{1}, x_{2}, \ldots, x_{n}\right\}$ be the finite universal set and let $A$, $B \in \operatorname{TzFIFS}(X)$ be given by

$$
\begin{gathered}
A=\left\{\left\langlex,\left[\mu_{A_{1}}(x), \mu_{A_{2}}(x), \mu_{A_{3}}(x), \mu_{A_{4}}(x)\right],\right.\right. \\
\left.\left.\left[\gamma_{A_{1}}(x), \gamma_{A_{2}}(x), \gamma_{A_{3}}(x), \gamma_{A_{4}}(x)\right]\right\rangle \times(x \in X)^{-1}\right\}, \\
B=\left\{\left\langlex,\left[\mu_{B_{1}}(x), \mu_{B_{2}}(x), \mu_{B_{3}}(x), \mu_{B_{4}}(x)\right],\right.\right. \\
\left.\left.\left[\gamma_{B_{1}}(x), \gamma_{B_{2}}(x), \gamma_{B_{3}}(x), \gamma_{B_{4}}(x)\right]\right\rangle \times(x \in X)^{-1}\right\},
\end{gathered}
$$

which are two trapezoidal fuzzy intuitionistic fuzzy numbers.

Then the correlation of trapezoidal fuzzy intuitionistic fuzzy numbers (TzFIFNs) is defined as follows.

Definition 3. Let $A=\left\{\left\langle x, \mu_{A}(x), \gamma_{A}(x)\right\rangle / x \in X\right\}$ be a trapezoidal fuzzy intuitionistic fuzzy number, where $\mu_{A}(x)=$ $\left(\mu_{A_{1}}(x), \mu_{A_{2}}(x), \mu_{A_{3}}(x), \mu_{A_{4}}(x)\right)$ is the membership degree, $\gamma_{A}(x)=\left(\gamma_{A_{1}}(x), \gamma_{A_{2}}(x), \gamma_{A_{3}}(x), \gamma_{A_{4}}(x)\right)$ is the nonmembership degree, and $\pi_{A}(x)=\left(\pi_{A_{1}}(x), \pi_{A_{2}}(x), \pi_{A_{3}}(x), \pi_{A_{4}}(x)\right)$ is the hesitation degree. Then the trapezoidal fuzzy intuitionistic energy of the sets $A$ and $B$ is defined as

$$
\begin{aligned}
& E_{\mathrm{TzFIFS}}(A) \\
& \begin{aligned}
=\frac{1}{4} \sum_{i=1}^{n}[ & \left.\mu_{A}^{2}\left(x_{i}\right)+\gamma_{A}^{2}\left(x_{i}\right)+\pi_{A}^{2}\left(x_{i}\right)\right] \\
=\frac{1}{4} \sum_{i=1}^{n}\{ & {\left[\left(\mu_{A_{1}}^{2}\left(x_{i}\right)+\mu_{A_{2}}^{2}\left(x_{i}\right)+\mu_{A_{3}}^{2}\left(x_{i}\right)+\mu_{A_{4}}^{2}\left(x_{i}\right)\right)\right] } \\
+ & {\left[\left(\gamma_{A_{1}}^{2}\left(x_{i}\right)+\gamma_{A_{2}}^{2}\left(x_{i}\right)+\gamma_{A_{3}}^{2}\left(x_{i}\right)+\gamma_{A_{4}}^{2}\left(x_{i}\right)\right)\right] } \\
+ & {\left.\left[\left(\pi_{A_{1}}^{2}\left(x_{i}\right)+\pi_{A_{2}}^{2}\left(x_{i}\right)+\pi_{A_{3}}^{2}\left(x_{i}\right)+\pi_{A_{4}}^{2}\left(x_{i}\right)\right)\right]\right\}, }
\end{aligned}
\end{aligned}
$$

$$
\begin{aligned}
& E_{\mathrm{TzFIFS}}(B) \\
&=\frac{1}{4} \sum_{i=1}^{n}\left[\mu_{B}^{2}\left(x_{i}\right)+\gamma_{B}^{2}\left(x_{i}\right)+\pi_{B}^{2}\left(x_{i}\right)\right] \\
&=\frac{1}{4} \sum_{i=1}^{n}\{[\left.\left(\mu_{B_{1}}^{2}\left(x_{i}\right)+\mu_{B_{2}}^{2}\left(x_{i}\right)+\mu_{B_{3}}^{2}\left(x_{i}\right)+\mu_{B_{4}}^{2}\left(x_{i}\right)\right)\right] \\
&+ {\left[\left(\gamma_{B_{1}}^{2}\left(x_{i}\right)+\gamma_{B_{2}}^{2}\left(x_{i}\right)+\gamma_{B_{3}}^{2}\left(x_{i}\right)+\gamma_{B_{4}}^{2}\left(x_{i}\right)\right)\right] } \\
&+ {\left.\left[\left(\pi_{B_{1}}^{2}\left(x_{i}\right)+\pi_{B_{2}}^{2}\left(x_{i}\right)+\pi_{B_{3}}^{2}\left(x_{i}\right)+\pi_{B_{4}}^{2}\left(x_{i}\right)\right)\right]\right\} . }
\end{aligned}
$$

Definition 4. Let $A=\left\{\left\langle x, \mu_{A}(x), \gamma_{A}(x)\right\rangle / x \in X\right\}$ be a trapezoidal fuzzy intuitionistic fuzzy number, where $\mu_{A}(x)=$ $\left(\mu_{A_{1}}(x), \mu_{A_{2}}(x), \mu_{A_{3}}(x), \mu_{A_{4}}(x)\right)$ is the membership degree, $\gamma_{A}(x)=\left(\gamma_{A_{1}}(x), \gamma_{A_{2}}(x), \gamma_{A_{3}}(x), \gamma_{A_{4}}(x)\right)$ is the nonmembership degree, and $\pi_{A}(x)=\left(\pi_{A_{1}}(x), \pi_{A_{2}}(x), \pi_{A_{3}}(x), \pi_{A_{4}}(x)\right)$ is 
the hesitation degree. Then the correlation between the sets $A$ and $B$ is defined as

$C_{\mathrm{TzFIFS}}(A, B)$

$$
\begin{gathered}
=\frac{1}{4} \sum_{i=1}^{n}\left[\mu_{A}\left(x_{i}\right) \mu_{B}\left(x_{i}\right)+\gamma_{A}\left(x_{i}\right) \gamma_{B}\left(x_{i}\right)+\pi_{A}\left(x_{i}\right) \pi_{B}\left(x_{i}\right)\right] \\
=\frac{1}{4} \sum_{i=1}^{n}\left\{\left[\left(\mu_{A_{1}}\left(x_{i}\right) \mu_{B_{1}}\left(x_{i}\right)+\mu_{A_{2}}\left(x_{i}\right) \mu_{B_{2}}\left(x_{i}\right)\right.\right.\right. \\
\left.\left.+\mu_{A_{3}}\left(x_{i}\right) \mu_{B_{3}}\left(x_{i}\right)+\mu_{A_{4}}\left(x_{i}\right) \mu_{B_{4}}\left(x_{i}\right)\right)\right] \\
+\left[\left(\gamma_{A_{1}}\left(x_{i}\right) \gamma_{B_{1}}\left(x_{i}\right)+\gamma_{A_{2}}\left(x_{i}\right) \gamma_{B_{2}}\left(x_{i}\right)\right.\right. \\
\left.\left.+\gamma_{A_{3}}\left(x_{i}\right) \gamma_{B_{3}}\left(x_{i}\right)+\gamma_{A_{4}}\left(x_{i}\right) \gamma_{B_{4}}\left(x_{i}\right)\right)\right] \\
+\left[\left(\pi_{A_{1}}\left(x_{i}\right) \pi_{B_{1}}\left(x_{i}\right)+\pi_{A_{2}}\left(x_{i}\right) \pi_{B_{2}}\left(x_{i}\right)\right.\right. \\
\left.\left.\left.+\pi_{A_{3}}\left(x_{i}\right) \pi_{B_{3}}\left(x_{i}\right)+\pi_{A_{4}}\left(x_{i}\right) \pi_{B_{4}}\left(x_{i}\right)\right)\right]\right\} .
\end{gathered}
$$

The correlation coefficient is given as

$$
\begin{array}{r}
K_{\mathrm{TzFIFS}}(A, B)=\frac{C_{\mathrm{TzFIFS}}(A, B)}{\sqrt{E_{\mathrm{TzFIFS}}(A) \cdot E_{\mathrm{TzFIFS}}(B)}}, \\
0 \leq K_{\mathrm{TzFIFS}}(A, B) \leq 1 .
\end{array}
$$

Proposition 5. For $A, B \in \operatorname{TzFIFS}(X)$ the following are true:

(1) $0 \leq K_{\text {TzFIFS }}(A, B) \leq 1$,

(2) $C_{\text {TzFIFS }}(A, B)=C_{\text {TzFIFS }}(B, A)$,

(3) $K_{\text {TzFIFS }}(A, B)=K_{\text {TzFIFS }}(B, A)$.

Theorem 6. For $A, B \in \operatorname{TzFIFS}(X)$, then $0 \leq K_{\text {TzFIFS }}(A, B) \leq$ 1.

Proof. Since $C_{\mathrm{TzFIFS}}(A, B) \geq 0$, it can be proved that $K_{\mathrm{TzFFF}}(A, B) \leq 1$. true:

For any arbitrary real number $\xi$, the following relation is

$$
\begin{aligned}
0 \leq \sum_{i=1}^{n} & \left\{\left(\mu_{A_{1}}\left(x_{i}\right)-\xi \mu_{B_{1}}\left(x_{i}\right)\right)^{2}+\left(\mu_{A_{2}}\left(x_{i}\right)-\xi \mu_{B_{2}}\left(x_{i}\right)\right)^{2}\right. \\
& +\left(\mu_{A_{3}}\left(x_{i}\right)-\xi \mu_{B_{3}}\left(x_{i}\right)\right)^{2}+\left(\mu_{A_{4}}\left(x_{i}\right)-\xi \mu_{B_{4}}\left(x_{i}\right)\right)^{2} \\
& +\left(\gamma_{A_{1}}\left(x_{i}\right)-\xi \gamma_{B_{1}}\left(x_{i}\right)\right)^{2}+\left(\gamma_{A_{2}}\left(x_{i}\right)-\xi \gamma_{B_{2}}\left(x_{i}\right)\right)^{2} \\
& +\left(\gamma_{A_{3}}\left(x_{i}\right)-\xi \gamma_{B_{3}}\left(x_{i}\right)\right)^{2}+\left(\gamma_{A_{4}}\left(x_{i}\right)-\xi \gamma_{B_{4}}\left(x_{i}\right)\right)^{2} \\
& +\left(\pi_{A_{1}}\left(x_{i}\right)-\xi \pi_{B_{1}}\left(x_{i}\right)\right)^{2}+\left(\pi_{A_{2}}\left(x_{i}\right)-\xi \pi_{B_{2}}\left(x_{i}\right)\right)^{2} \\
& \left.+\left(\pi_{A_{3}}\left(x_{i}\right)-\xi \pi_{B_{3}}\left(x_{i}\right)\right)^{2}+\left(\pi_{A_{4}}\left(x_{i}\right)-\xi \pi_{B_{4}}\left(x_{i}\right)\right)^{2}\right\}
\end{aligned}
$$

$$
\begin{aligned}
=\sum_{i=1}^{n}\left\{\left(\mu_{A_{1}}^{2}\left(x_{i}\right)+\gamma_{A_{1}}^{2}\left(x_{i}\right)+\pi_{A_{1}}^{2}\left(x_{i}\right)\right)\right. \\
+ \\
+\left(\mu_{A_{2}}^{2}\left(x_{i}\right)+\gamma_{A_{2}}^{2}\left(x_{i}\right)+\pi_{A_{2}}^{2}\left(x_{i}\right)\right) \\
+\left(\mu_{A_{3}}^{2}\left(x_{i}\right)+\gamma_{A_{3}}^{2}\left(x_{i}\right)+\pi_{A_{3}}^{2}\left(x_{i}\right)\right) \\
+\quad\left(\mu_{A_{4}}^{2}\left(x_{i}\right)+\gamma_{A_{4}}^{2}\left(x_{i}\right)+\pi_{A_{4}}^{2}\left(x_{i}\right)\right) \\
-2 \xi\left(\mu_{A_{1}}\left(x_{i}\right) \mu_{B_{1}}\left(x_{i}\right)+\gamma_{A_{1}}\left(x_{i}\right) \gamma_{B_{1}}\left(x_{i}\right)\right. \\
\left.+\pi_{A_{1}}\left(x_{i}\right) \pi_{B_{1}}\left(x_{i}\right)\right) \\
-2 \xi\left(\mu_{A_{2}}\left(x_{i}\right) \mu_{B_{2}}\left(x_{i}\right)+\gamma_{A_{2}}\left(x_{i}\right) \gamma_{B_{2}}\left(x_{i}\right)\right. \\
\left.+\pi_{A_{2}}\left(x_{i}\right) \pi_{B_{2}}\left(x_{i}\right)\right) \\
-2 \xi\left(\mu_{A_{3}}\left(x_{i}\right) \mu_{B_{3}}\left(x_{i}\right)+\gamma_{A_{3}}\left(x_{i}\right) \gamma_{B_{3}}\left(x_{i}\right)\right. \\
\left.+\pi_{A_{3}}\left(x_{i}\right) \pi_{B_{3}}\left(x_{i}\right)\right) \\
+2 \xi\left(\mu_{A_{4}}\left(x_{i}\right) \mu_{B_{4}}\left(x_{i}\right)+\gamma_{A_{4}}\left(x_{i}\right) \gamma_{B_{4}}\left(x_{i}\right)\right. \\
\left.+\pi_{A_{4}}\left(x_{i}\right) \pi_{B_{4}}\left(x_{i}\right)\right) \\
+\xi^{2}\left(\mu_{B_{1}}^{2}\left(x_{i}\right)+\gamma_{B_{1}}^{2}\left(x_{i}\right)+\pi_{B_{1}}^{2}\left(x_{i}\right)\right) \\
+\xi^{2}\left(\mu_{B_{2}}^{2}\left(x_{i}\right)+\gamma_{B_{2}}^{2}\left(x_{i}\right)+\pi_{B_{2}}^{2}\left(x_{i}\right)\right) \\
+\xi^{2}\left(\mu_{B_{3}}^{2}\left(x_{i}\right)+\gamma_{B_{3}}^{2}\left(x_{i}\right)+\pi_{B_{3}}^{2}\left(x_{i}\right)\right) \\
\left.+\xi^{2}\left(\mu_{B_{4}}^{2}\left(x_{i}\right)+\gamma_{B_{4}}^{2}\left(x_{i}\right)+\pi_{B_{4}}^{2}\left(x_{i}\right)\right)\right\} .
\end{aligned}
$$

Hence,

$$
\begin{gathered}
\left\{\sum _ { i = 1 } ^ { n } \left[\left(\mu_{A_{1}}\left(x_{i}\right) \mu_{B_{1}}\left(x_{i}\right)+\mu_{A_{2}}\left(x_{i}\right) \mu_{B_{2}}\left(x_{i}\right)\right.\right.\right. \\
+\mu_{A_{3}}\left(x_{i}\right) \mu_{B_{3}}\left(x_{i}\right)+\mu_{A_{4}}\left(x_{i}\right) \mu_{B_{4}}\left(x_{i}\right) \\
+\gamma_{A_{1}}\left(x_{i}\right) \gamma_{B_{1}}\left(x_{i}\right)+\gamma_{A_{2}}\left(x_{i}\right) \gamma_{B_{2}}\left(x_{i}\right) \\
+\gamma_{A_{3}}\left(x_{i}\right) \gamma_{B_{3}}\left(x_{i}\right)+\gamma_{A_{4}}\left(x_{i}\right) \gamma_{B_{4}}\left(x_{i}\right) \\
+\pi_{A_{1}}\left(x_{i}\right) \pi_{B_{1}}\left(x_{i}\right)+\pi_{A_{2}}\left(x_{i}\right) \pi_{B_{2}}\left(x_{i}\right) \\
\left.\left.\left.+\pi_{A_{3}}\left(x_{i}\right) \pi_{B_{3}}\left(x_{i}\right)+\pi_{A_{4}}\left(x_{i}\right) \pi_{B_{4}}\left(x_{i}\right)\right)\right]\right\}^{2} \\
\leq\left(\sum _ { i = 1 } ^ { n } \left\{\left(\mu_{A_{1}}^{2}\left(x_{i}\right)+\gamma_{A_{1}}^{2}\left(x_{i}\right)+\pi_{A_{1}}^{2}\left(x_{i}\right)\right)\right.\right. \\
+\left(\mu_{A_{2}}^{2}\left(x_{i}\right)+\gamma_{A_{2}}^{2}\left(x_{i}\right)+\pi_{A_{2}}^{2}\left(x_{i}\right)\right) \\
+\left(\mu_{A_{3}}^{2}\left(x_{i}\right)+\gamma_{A_{3}}^{2}\left(x_{i}\right)+\pi_{A_{3}}^{2}\left(x_{i}\right)\right) \\
\left.+\left(\mu_{A_{4}}^{2}\left(x_{i}\right)+\gamma_{A_{4}}^{2}\left(x_{i}\right)+\pi_{A_{4}}^{2}\left(x_{i}\right)\right)\right\}
\end{gathered}
$$




$$
\begin{aligned}
\times \sum_{i=1}^{n}\{ & \left(\mu_{B_{1}}^{2}\left(x_{i}\right)+\gamma_{B_{1}}^{2}\left(x_{i}\right)+\pi_{B_{1}}^{2}\left(x_{i}\right)\right) \\
& +\left(\mu_{B_{2}}^{2}\left(x_{i}\right)+\gamma_{B_{2}}^{2}\left(x_{i}\right)+\pi_{B_{2}}^{2}\left(x_{i}\right)\right) \\
& \left.+\left(\mu_{B_{3}}^{2}\left(x_{i}\right)+\gamma_{B_{3}}^{2}\left(x_{i}\right)+\pi_{B_{3}}^{2}\left(x_{i}\right)\right)\right\} \\
& \left.+\left(\mu_{B_{4}}^{2}\left(x_{i}\right)+\gamma_{B_{4}}^{2}\left(x_{i}\right)+\pi_{B_{4}}^{2}\left(x_{i}\right)\right)\right) .
\end{aligned}
$$

The above inequality can be written as

$$
\begin{aligned}
& \left(\left\{\sum _ { i = 1 } ^ { n } \left[\mu_{A_{1}}\left(x_{i}\right) \mu_{B_{1}}\left(x_{i}\right)+\mu_{A_{2}}\left(x_{i}\right) \mu_{B_{2}}\left(x_{i}\right)\right.\right.\right. \\
& +\mu_{A_{3}}\left(x_{i}\right) \mu_{B_{3}}\left(x_{i}\right)+\mu_{A_{4}}\left(x_{i}\right) \mu_{B_{4}}\left(x_{i}\right) \\
& +\gamma_{A_{1}}\left(x_{i}\right) \gamma_{B_{1}}\left(x_{i}\right)+\gamma_{A_{2}}\left(x_{i}\right) \gamma_{B_{2}}\left(x_{i}\right) \\
& +\gamma_{A_{3}}\left(x_{i}\right) \gamma_{B_{3}}\left(x_{i}\right)+\gamma_{A_{4}}\left(x_{i}\right) \gamma_{B_{4}}\left(x_{i}\right) \\
& +\pi_{A_{1}}\left(x_{i}\right) \pi_{B_{1}}\left(x_{i}\right)+\pi_{A_{2}}\left(x_{i}\right) \pi_{B_{2}}\left(x_{i}\right) \\
& \left.\left.+\pi_{A_{3}}\left(x_{i}\right) \pi_{B_{3}}\left(x_{i}\right)+\pi_{A_{4}}\left(x_{i}\right) \pi_{B_{4}}\left(x_{i}\right)\right]^{2}\right\} \\
& \times\left(\left(\sum _ { i = 1 } ^ { n } \left\{\left(\mu_{A_{1}}^{2}\left(x_{i}\right)+\gamma_{A_{1}}^{2}\left(x_{i}\right)+\pi_{A_{1}}^{2}\left(x_{i}\right)\right)\right.\right.\right. \\
& +\left(\mu_{A_{2}}^{2}\left(x_{i}\right)+\gamma_{A_{2}}^{2}\left(x_{i}\right)+\pi_{A_{2}}^{2}\left(x_{i}\right)\right) \\
& +\left(\mu_{A_{3}}^{2}\left(x_{i}\right)+\gamma_{A_{3}}^{2}\left(x_{i}\right)+\pi_{A_{3}}^{2}\left(x_{i}\right)\right) \\
& \left.+\left(\mu_{A_{4}}^{2}\left(x_{i}\right)+\gamma_{A_{4}}^{2}\left(x_{i}\right)+\pi_{A_{4}}^{2}\left(x_{i}\right)\right)\right\} \\
& \times \sum_{i=1}^{n}\left\{\left(\mu_{B_{1}}^{2}\left(x_{i}\right)+\gamma_{B_{1}}^{2}\left(x_{i}\right)+\pi_{B_{1}}^{2}\left(x_{i}\right)\right)\right. \\
& +\left(\mu_{B_{2}}^{2}\left(x_{i}\right)+\gamma_{B_{2}}^{2}\left(x_{i}\right)+\pi_{B_{2}}^{2}\left(x_{i}\right)\right) \\
& +\left(\mu_{B_{3}}^{2}\left(x_{i}\right)+\gamma_{B_{3}}^{2}\left(x_{i}\right)+\pi_{B_{3}}^{2}\left(x_{i}\right)\right) \\
& \left.\left.\left.\left.+\left(\mu_{B_{4}}^{2}\left(x_{i}\right)+\gamma_{B_{4}}^{2}\left(x_{i}\right)+\pi_{B_{4}}^{2}\left(x_{i}\right)\right)\right\}\right)\right)^{-1}\right) .
\end{aligned}
$$

Therefore $\left[C_{\mathrm{TzFIFS}}(A, B)\right]^{2} / E_{\mathrm{TzFIFS}}(A) \cdot E_{\mathrm{TzFIFS}}(B) \leq 1$; hence $K_{\mathrm{TzFIFS}}(A, B)=C_{\mathrm{TzFIFS}}(A, B) / \sqrt{E_{\mathrm{TzFIFS}}(A) \cdot E_{\mathrm{TzFIFS}}(B)} \leq 1$.

Theorem 7. Consider $K_{\text {TzFIFS }}(A, B)=1 \Leftrightarrow A=B$.

Proof. Considering the inequality in the proof of Theorem 6, then the equality holds if and only if the following are satisfied (i) $\mu_{A_{1}}\left(x_{i}\right)=\xi \mu_{B_{1}}\left(x_{i}\right), \mu_{A_{2}}\left(x_{i}\right)=\xi \mu_{B_{2}}\left(x_{i}\right), \mu_{A_{3}}\left(x_{i}\right)=$ $\xi \mu_{B_{3}}\left(x_{i}\right), \mu_{A_{4}}\left(x_{i}\right)=\xi \mu_{B_{4}}\left(x_{i}\right)$,

(ii) $\gamma_{A_{1}}\left(x_{i}\right)=\xi \gamma_{B_{1}}\left(x_{i}\right), \gamma_{A_{2}}\left(x_{i}\right)=\xi \gamma_{B_{2}}\left(x_{i}\right), \gamma_{A_{3}}\left(x_{i}\right)=$ $\xi \gamma_{B_{3}}\left(x_{i}\right), \gamma_{A_{4}}\left(x_{i}\right)=\xi \gamma_{B_{4}}\left(x_{i}\right)$

(iii) $\pi_{A_{1}}\left(x_{i}\right)=\xi \pi_{B_{1}}\left(x_{i}\right), \pi_{A_{2}}\left(x_{i}\right)=\xi \pi_{B_{2}}\left(x_{i}\right), \pi_{A_{3}}\left(x_{i}\right)=$ $\xi \pi_{B_{3}}\left(x_{i}\right), \pi_{A_{4}}\left(x_{i}\right)=\xi \pi_{B_{4}}\left(x_{i}\right)$

for some positive real $\xi$.

As

$$
\begin{aligned}
& \mu_{A_{1}}\left(x_{i}\right)+\gamma_{A_{1}}\left(x_{i}\right)+\pi_{A_{1}}\left(x_{i}\right) \\
& =\mu_{B_{1}}\left(x_{i}\right)+\gamma_{B_{1}}\left(x_{i}\right)+\pi_{B_{1}}\left(x_{i}\right)=1, \\
& \mu_{A_{2}}\left(x_{i}\right)+\gamma_{A_{2}}\left(x_{i}\right)+\pi_{A_{2}}\left(x_{i}\right) \\
& =\mu_{B_{2}}\left(x_{i}\right)+\gamma_{B_{2}}\left(x_{i}\right)+\pi_{B_{2}}\left(x_{i}\right)=1, \\
& \mu_{A_{3}}\left(x_{i}\right)+\gamma_{A_{3}}\left(x_{i}\right)+\pi_{A_{3}}\left(x_{i}\right) \\
& =\mu_{B_{3}}\left(x_{i}\right)+\gamma_{B_{3}}\left(x_{i}\right)+\pi_{B_{3}}\left(x_{i}\right)=1, \\
& \mu_{A_{4}}\left(x_{i}\right)+\gamma_{A_{4}}\left(x_{i}\right)+\pi_{A_{4}}\left(x_{i}\right) \\
& =\mu_{B_{4}}\left(x_{i}\right)+\gamma_{B_{4}}\left(x_{i}\right)+\pi_{B_{4}}\left(x_{i}\right)=1,
\end{aligned}
$$

then it means $\xi=1$, and therefore $A=B$.

Theorem 8. $C_{\text {TrFIFS }}(A, B)=0 \Leftrightarrow A$ and $B$ are nonfuzzy sets and satisfy the condition $\mu_{A}\left(x_{i}\right)+\mu_{B}\left(x_{i}\right)=1$ or $\gamma_{A}\left(x_{i}\right)+$ $\gamma_{B}\left(x_{i}\right)=1$ or $\pi_{A}\left(x_{i}\right)+\pi_{B}\left(x_{i}\right)=1$, for all $x_{i} \in X$.

Proof. For all $x_{i} \in X$, the following are true:

$$
\begin{aligned}
& \left(\mu_{A_{1}}\left(x_{i}\right) \mu_{B_{1}}\left(x_{i}\right)+\gamma_{A_{1}}\left(x_{i}\right) \gamma_{B_{1}}\left(x_{i}\right)\right. \\
& \left.+\pi_{A_{1}}\left(x_{i}\right) \pi_{B_{1}}\left(x_{i}\right)\right) \geq 0, \\
& \left(\mu_{A_{2}}\left(x_{i}\right) \mu_{B_{2}}\left(x_{i}\right)+\gamma_{A_{2}}\left(x_{i}\right) \gamma_{B_{2}}\left(x_{i}\right)\right. \\
& \left.+\pi_{A_{2}}\left(x_{i}\right) \pi_{B_{2}}\left(x_{i}\right)\right) \geq 0, \\
& \left(\mu_{A_{3}}\left(x_{i}\right) \mu_{B_{3}}\left(x_{i}\right)+\gamma_{A_{3}}\left(x_{i}\right) \gamma_{B_{3}}\left(x_{i}\right)\right. \\
& \left.+\pi_{A_{3}}\left(x_{i}\right) \pi_{B_{3}}\left(x_{i}\right)\right) \geq 0, \\
& \left(\mu_{A_{4}}\left(x_{i}\right) \mu_{B_{4}}\left(x_{i}\right)+\gamma_{A_{4}}\left(x_{i}\right) \gamma_{B_{4}}\left(x_{i}\right)\right. \\
& \left.+\pi_{A_{4}}\left(x_{i}\right) \pi_{B_{4}}\left(x_{i}\right)\right) \geq 0 .
\end{aligned}
$$


Hence,

$$
\begin{aligned}
& \left\{\left(\mu_{A_{1}}\left(x_{i}\right) \mu_{B_{1}}\left(x_{i}\right)+\gamma_{A_{1}}\left(x_{i}\right) \gamma_{B_{1}}\left(x_{i}\right)\right.\right. \\
& \left.+\pi_{A_{1}}\left(x_{i}\right) \pi_{B_{1}}\left(x_{i}\right)\right) \\
& +\left(\mu_{A_{2}}\left(x_{i}\right) \mu_{B_{2}}\left(x_{i}\right)+\gamma_{A_{2}}\left(x_{i}\right) \gamma_{B_{2}}\left(x_{i}\right)\right. \\
& \left.+\pi_{A_{2}}\left(x_{i}\right) \pi_{B_{2}}\left(x_{i}\right)\right) \\
& +\left(\mu_{A_{3}}\left(x_{i}\right) \mu_{B_{3}}\left(x_{i}\right)+\gamma_{A_{3}}\left(x_{i}\right) \gamma_{B_{3}}\left(x_{i}\right)\right. \\
& \left.+\pi_{A_{3}}\left(x_{i}\right) \pi_{B_{3}}\left(x_{i}\right)\right) \\
& +\left(\mu_{A_{4}}\left(x_{i}\right) \mu_{B_{4}}\left(x_{i}\right)+\gamma_{A_{4}}\left(x_{i}\right) \gamma_{B_{4}}\left(x_{i}\right)\right. \\
& \left.\left.+\pi_{A_{4}}\left(x_{i}\right) \pi_{B_{4}}\left(x_{i}\right)\right)\right\} \geq 0, \\
& \left\{\mu_{A_{1}}\left(x_{i}\right) \mu_{B_{1}}\left(x_{i}\right)+\mu_{A_{2}}\left(x_{i}\right) \mu_{B_{2}}\left(x_{i}\right)\right. \\
& +\mu_{A_{3}}\left(x_{i}\right) \mu_{B_{3}}\left(x_{i}\right)+\mu_{A_{4}}\left(x_{i}\right) \mu_{B_{4}}\left(x_{i}\right) \\
& +\gamma_{A_{1}}\left(x_{i}\right) \gamma_{B_{1}}\left(x_{i}\right)+\gamma_{A_{2}}\left(x_{i}\right) \gamma_{B_{2}}\left(x_{i}\right) \\
& +\gamma_{A_{3}}\left(x_{i}\right) \gamma_{B_{3}}\left(x_{i}\right)+\gamma_{A_{4}}\left(x_{i}\right) \gamma_{B_{4}}\left(x_{i}\right) \\
& +\pi_{A_{1}}\left(x_{i}\right) \pi_{B_{1}}\left(x_{i}\right)+\pi_{A_{2}}\left(x_{i}\right) \pi_{B_{2}}\left(x_{i}\right) \\
& \left.+\pi_{A_{3}}\left(x_{i}\right) \pi_{B_{3}}\left(x_{i}\right)+\pi_{A_{4}}\left(x_{i}\right) \pi_{B_{4}}\left(x_{i}\right)\right\} \geq 0 .
\end{aligned}
$$

If $C_{\mathrm{TzFIFS}}(A, B)=0$ for all $x_{i} \in X$, then the following should be true:

$$
\begin{aligned}
& \mu_{A_{1}}\left(x_{i}\right) \mu_{B_{1}}\left(x_{i}\right)+\mu_{A_{2}}\left(x_{i}\right) \mu_{B_{2}}\left(x_{i}\right) \\
& \quad+\mu_{A_{3}}\left(x_{i}\right) \mu_{B_{3}}\left(x_{i}\right)+\mu_{A_{4}}\left(x_{i}\right) \mu_{B_{4}}\left(x_{i}\right)=0, \\
& \gamma_{A_{1}}\left(x_{i}\right) \gamma_{B_{1}}\left(x_{i}\right)+\gamma_{A_{2}}\left(x_{i}\right) \gamma_{B_{2}}\left(x_{i}\right) \\
& \quad+\gamma_{A_{3}}\left(x_{i}\right) \gamma_{B_{3}}\left(x_{i}\right)+\gamma_{A_{4}}\left(x_{i}\right) \gamma_{B_{4}}\left(x_{i}\right)=0, \\
& \pi_{A_{1}}\left(x_{i}\right) \pi_{B_{1}}\left(x_{i}\right)+\pi_{A_{2}}\left(x_{i}\right) \pi_{B_{2}}\left(x_{i}\right) \\
& \quad+\pi_{A_{3}}\left(x_{i}\right) \pi_{B_{3}}\left(x_{i}\right)+\pi_{A_{4}}\left(x_{i}\right) \pi_{B_{4}}\left(x_{i}\right)=0 .
\end{aligned}
$$

(i) If $\mu_{A_{1}}\left(x_{i}\right)=1$ then $\mu_{B_{1}}\left(x_{i}\right)=0$ and $\gamma_{A_{1}}\left(x_{i}\right)=$ $\pi_{A_{1}}\left(x_{i}\right)=0$,

(ii) if $\mu_{A_{2}}\left(x_{i}\right)=1$ then $\mu_{B_{2}}\left(x_{i}\right)=0$ and $\gamma_{A_{2}}\left(x_{i}\right)=$ $\pi_{A_{2}}\left(x_{i}\right)=0$,

(iii) if $\mu_{A_{3}}\left(x_{i}\right)=1$ then $\mu_{B_{3}}\left(x_{i}\right)=0$ and $\gamma_{A_{3}}\left(x_{i}\right)=$ $\pi_{A_{3}}\left(x_{i}\right)=0$,

(iv) if $\mu_{A_{4}}\left(x_{i}\right)=1$ then $\mu_{B_{4}}\left(x_{i}\right)=0$ and $\gamma_{A_{4}}\left(x_{i}\right)=$ $\pi_{A_{4}}\left(x_{i}\right)=0$.

Also,

(i) if $\mu_{B_{1}}\left(x_{i}\right)=1$ then $\mu_{A_{1}}\left(x_{i}\right)=0$ and $\gamma_{B_{1}}\left(x_{i}\right)=$ $\pi_{B_{1}}\left(x_{i}\right)=0$,

(ii) if $\mu_{B_{2}}\left(x_{i}\right)=1$ then $\mu_{A_{2}}\left(x_{i}\right)=0$ and $\gamma_{B_{2}}\left(x_{i}\right)=$ $\pi_{B_{2}}\left(x_{i}\right)=0$ (iii) if $\mu_{B_{3}}\left(x_{i}\right)=1$ then $\mu_{A_{3}}\left(x_{i}\right)=0$ and $\gamma_{B_{3}}\left(x_{i}\right)=$ $\pi_{B_{3}}\left(x_{i}\right)=0$,

(iv) if $\mu_{B_{4}}\left(x_{i}\right)=1$ then $\mu_{A_{4}}\left(x_{i}\right)=0$ and $\gamma_{B_{4}}\left(x_{i}\right)=$ $\pi_{B_{4}}\left(x_{i}\right)=0$.

Hence $\mu_{A_{1}}\left(x_{i}\right)+\mu_{B_{1}}\left(x_{i}\right)=1, \mu_{A_{2}}\left(x_{i}\right)+\mu_{B_{2}}\left(x_{i}\right)=1$, $\mu_{A_{3}}\left(x_{i}\right)+\mu_{B_{3}}\left(x_{i}\right)=1$, and $\mu_{A_{4}}\left(x_{i}\right)+\mu_{B_{4}}\left(x_{i}\right)=1$.

Conversely, when $A$ and $B$ are nonfuzzy sets and $\mu_{A_{1}}\left(x_{i}\right)+$ $\mu_{B_{1}}\left(x_{i}\right)=1, \mu_{A_{2}}\left(x_{i}\right)+\mu_{B_{2}}\left(x_{i}\right)=1, \mu_{A_{3}}\left(x_{i}\right)+\mu_{B_{3}}\left(x_{i}\right)=$ 1 , and $\mu_{A_{4}}\left(x_{i}\right)+\mu_{B_{4}}\left(x_{i}\right)=1$.

If $\mu_{A_{1}}\left(x_{i}\right)=1$ then $\mu_{B_{1}}\left(x_{i}\right)=0$ and $\gamma_{A_{1}}\left(x_{i}\right)=\pi_{A_{1}}\left(x_{i}\right)=0$.

This property can be observed similarly for all other entries. Therefore $C_{\text {TrFIFS }}(A, B)=0$.

The cases $\gamma_{A}\left(x_{i}\right)+\gamma_{B}\left(x_{i}\right)=1$ and $\pi_{A}\left(x_{i}\right)+\pi_{B}\left(x_{i}\right)=1$ can be proved similarly.

Theorem 9. $C_{T z F I F S}(A, A)=1 \Leftrightarrow A$ is a nonfuzzy set.

Proof. If $A$ is a nonfuzzy set, then $C_{\mathrm{TzFIFS}}(A, A)=1$ is obvious. tion.

Conversely, it can be proved by the method of contradic-

Assume $A$ is not a nonfuzzy set.

Then $0 \leq \mu_{A}\left(x_{i}\right)<1,0 \leq \gamma_{A}\left(x_{i}\right)<1$, and $0 \leq \pi_{A}\left(x_{i}\right)<1$, for some $x_{i}$.

Hence $\mu_{A}^{2}\left(x_{i}\right)+\gamma_{A}^{2}\left(x_{i}\right)+\pi_{A}^{2}\left(x_{i}\right)<1$.

That is,

$$
\begin{aligned}
& \mu_{A_{1}}^{2}\left(x_{i}\right)+\gamma_{A_{1}}^{2}\left(x_{i}\right)+\pi_{A_{1}}^{2}\left(x_{i}\right)<1, \\
& \mu_{A_{2}}^{2}\left(x_{i}\right)+\gamma_{A_{2}}^{2}\left(x_{i}\right)+\pi_{A_{2}}^{2}\left(x_{i}\right)<1, \\
& \mu_{A_{3}}^{2}\left(x_{i}\right)+\gamma_{A_{3}}^{2}\left(x_{i}\right)+\pi_{A_{3}}^{2}\left(x_{i}\right)<1, \\
& \mu_{A_{4}}^{2}\left(x_{i}\right)+\gamma_{A_{4}}^{2}\left(x_{i}\right)+\pi_{A_{4}}^{2}\left(x_{i}\right)<1 .
\end{aligned}
$$

Also,

$$
\begin{aligned}
& \left\{\mu_{A_{1}}^{2}\left(x_{i}\right)+\mu_{A_{2}}^{2}\left(x_{i}\right)+\mu_{A_{3}}^{2}\left(x_{i}\right)+\mu_{A_{4}}^{2}\left(x_{i}\right)\right. \\
& \quad+\gamma_{A_{1}}^{2}\left(x_{i}\right)+\gamma_{A_{2}}^{2}\left(x_{i}\right)+\gamma_{A_{3}}^{2}\left(x_{i}\right)+\gamma_{A_{4}}^{2}\left(x_{i}\right) \\
& \left.\quad+\pi_{A_{1}}^{2}\left(x_{i}\right)+\pi_{A_{2}}^{2}\left(x_{i}\right)+\pi_{A_{3}}^{2}\left(x_{i}\right)+\pi_{A_{4}}^{2}\left(x_{i}\right)\right\}<1 .
\end{aligned}
$$

Then

$$
\begin{aligned}
C_{\mathrm{TzFIFS}}(A, A)=\frac{1}{3} \sum_{i=1}^{n}\{ & \mu_{A_{1}}^{2}\left(x_{i}\right)+\mu_{A_{2}}^{2}\left(x_{i}\right)+\mu_{A_{3}}^{2}\left(x_{i}\right) \\
& +\mu_{A_{4}}^{2}\left(x_{i}\right)+\gamma_{A_{1}}^{2}\left(x_{i}\right)+\gamma_{A_{2}}^{2}\left(x_{i}\right) \\
& +\gamma_{A_{3}}^{2}\left(x_{i}\right)+\gamma_{A_{4}}^{2}\left(x_{i}\right) \\
& +\pi_{A_{1}}^{2}\left(x_{i}\right)+\pi_{A_{2}}^{2}\left(x_{i}\right) \\
& \left.+\pi_{A_{3}}^{2}\left(x_{i}\right)+\pi_{A_{4}}^{2}\left(x_{i}\right)\right\}<1 .
\end{aligned}
$$

This is contradictory, and so $A$ is a nonfuzzy set. 


\section{WA and WG Operators for TzFIFNs}

On the foundation of the definitions discussed by Wang [13], the weighted arithmetic operators for TzFIFNs are given as follows.

Definition 10. Let $\tilde{a}_{j}=\left\langle\left[\mu_{1 j}, \mu_{2 j}, \mu_{3 j}, \mu_{4 j}\right],\left[\gamma_{1 j}, \gamma_{2 j}, \gamma_{3 j}, \gamma_{4 j}\right]\right\rangle$, $j=1,2, \ldots, n$, be a collection of TzFIFN values. The trapezoidal fuzzy intuitionistic fuzzy weighted averaging (TzFIFWA) operator, TzFIFWA : $Q^{n} \rightarrow Q$, is defined as

$$
\begin{aligned}
& \operatorname{TzFIFWA}_{\omega}\left(\tilde{a}_{1}, \tilde{a}_{2}, \ldots, \tilde{a}_{n}\right) \\
&= \sum_{j=1}^{n} \omega_{j} \tilde{a}_{j}=\left(1-\prod_{j=1}^{n}\left(1-\mu_{j}\right)^{\omega_{j}}, \prod_{j=1}^{n}\left(\gamma_{j}\right)^{\omega_{j}}\right) \\
&= {\left[1-\prod_{j=1}^{n}\left(1-\mu_{1 j}\right)^{\omega_{j}},\right.} \\
& 1-\prod_{j=1}^{n}\left(1-\mu_{2 j}\right)^{\omega_{j}}, 1-\prod_{j=1}^{n}\left(1-\mu_{3 j}\right)^{\omega_{j}}, \\
&\left.1-\prod_{j=1}^{n}\left(1-\mu_{4 j}\right)^{\omega_{j}}\right], \\
& {\left[\prod_{j=1}^{n}\left(\gamma_{1 j}\right)^{\omega_{j}}, \prod_{j=1}^{n}\left(\gamma_{2 j}\right)^{\omega_{j}},\right.} \\
&\left.\left.\prod_{j=1}^{n}\left(\gamma_{3 j}\right)^{\omega_{j}}, \prod_{j=1}^{n}\left(\gamma_{4 j}\right)^{\omega_{j}}\right]\right),
\end{aligned}
$$

where $\omega=\left(\omega_{1}, \omega_{2}, \ldots, \omega_{t}\right)^{T}$ is the weight vector of $\widetilde{a}_{j}=$ $\left\langle\left[\mu_{1 j}, \mu_{2 j}, \mu_{3 j}, \mu_{4 j}\right],\left[\gamma_{1 j}, \gamma_{2 j}, \gamma_{3 j}, \gamma_{4 j}\right]\right\rangle$, such that $\omega_{j}>0$ and $\sum_{j=1}^{n} \omega_{j}=1$.

Definition 11. Let $\tilde{a}_{j}=\left\langle\left[\mu_{1 j}, \mu_{2 j}, \mu_{3 j}, \mu_{4 j}\right],\left[\gamma_{1 j}, \gamma_{2 j}, \gamma_{3 j}, \gamma_{4 j}\right]\right\rangle$, $j=1,2, \ldots, n$, be a collection of TzFIFN values. The trapezoidal fuzzy intuitionistic fuzzy weighted geometric (TzFIFWG) operator, TzFIFWG : $Q^{n} \rightarrow Q$, is defined as

$$
\begin{aligned}
& \operatorname{TzFIFWG}_{\omega}\left(\tilde{a}_{1}, \tilde{a}_{2}, \ldots, \tilde{a}_{n}\right) \\
& =\sum_{j=1}^{n} \omega_{j} \tilde{a}_{j}=\left(\prod_{j=1}^{n}\left(\mu_{j}\right)^{\omega_{j}}, 1-\prod_{j=1}^{n}\left(1-\gamma_{j}\right)^{\omega_{j}}\right) \\
& =\left(\left[\prod_{j=1}^{n}\left(\mu_{1 j}\right)^{\omega_{j}}, \prod_{j=1}^{n}\left(\mu_{2 j}\right)^{\omega_{j}},\right.\right. \\
& \left.\prod_{j=1}^{n}\left(\mu_{3 j}\right)^{\omega_{j}}, \prod_{j=1}^{n}\left(\mu_{4 j}\right)^{\omega_{j}}\right],
\end{aligned}
$$

$$
\begin{aligned}
& {\left[1-\prod_{j=1}^{n}\left(1-\gamma_{1 j}\right)^{\omega_{j}}, 1-\prod_{j=1}^{n}\left(1-\gamma_{2 j}\right)^{\omega_{j}},\right.} \\
& \left.\left.1-\prod_{j=1}^{n}\left(1-\gamma_{3 j}\right)^{\omega_{j}}, 1-\prod_{j=1}^{n}\left(1-\gamma_{4 j}\right)^{\omega_{j}}\right]\right),
\end{aligned}
$$

where $\omega=\left(\omega_{1}, \omega_{2}, \ldots, \omega_{t}\right)^{T}$ is the weight vector of $\tilde{a}_{j}=$ $\left\langle\left[\mu_{1 j}, \mu_{2 j}, \mu_{3 j}, \mu_{4 j}\right],\left[\gamma_{1 j}, \gamma_{2 j}, \gamma_{3 j}, \gamma_{4 j}\right]\right\rangle$, such that $\omega_{j}>0$ and $\sum_{j=1}^{n} \omega_{j}=1$.

\section{MADM Algorithm for Trapezoidal Fuzzy IFS}

Let $A=\left\{A_{1}, A_{2}, \ldots, A_{m}\right\}$ be a set of alternatives, and let $G=$ $\left\{G_{1}, G_{2}, \ldots, G_{n}\right\}$ be the set of attributes; $\omega=\left(\omega_{1}, \omega_{2}, \ldots, \omega_{n}\right)$ is the weighting vector of the attribute $G_{j}, j=1,2, \ldots, n$, where $\omega_{j} \in[0,1]$, such that $\sum_{j=1}^{n} \omega_{j}=1$. Suppose that

$$
\begin{gathered}
\widetilde{R}_{k}=\left(\widetilde{r}_{h i j}^{(k)}\right)_{m \times n}=\left(\mu_{h i j}^{(k)}, \gamma_{h i j}^{(k)}\right)_{m \times n} \\
=\left(\left[\mu_{1 i j}^{(k)}, \mu_{2 i j}^{(k)}, \mu_{3 i j}^{(k)}, \mu_{4 i j}^{(k)}\right],\right. \\
\left.\left[\gamma_{1 i j}^{(k)}, \gamma_{2 i j}^{(k)}, \gamma_{3 i j}^{(k)}, \gamma_{4 i j}^{(k)}\right]\right)_{m \times n}
\end{gathered}
$$

be the trapezoidal fuzzy intuitionistic fuzzy number decision matrix, where $\left[\mu_{1 i j}^{(k)}, \mu_{2 i j}^{(k)}, \mu_{3 i j}^{(k)}, \mu_{4 i j}^{(k)}\right]$ is the degree of the membership value that the alternative $A_{i}$ satisfies the attribute $G_{j}$ given by the decision maker $D_{k}$ and $\left[\gamma_{1 i j}^{(k)}, \gamma_{2 i j}^{(k)}, \gamma_{3 i j}^{(k)}, \gamma_{4 i j}^{(k)}\right]$ is the degree of nonmembership value for the alternative $A_{i}$, where $\mu_{h i j}^{(k)}, \gamma_{h i j}^{(k)} \subset[0,1]$ and $\mu_{4 i j}^{(k)}, \gamma_{4 i j}^{(k)} \leq 1, i=1,2, \ldots, m$, $j=1,2, \ldots, n$, and $k=1,2, \ldots, t$.

The developed model of MADM is given as follows.

Step 1. Utilize the decision information given in the matrix $\widetilde{R}$ and the TzFIFWA or TzFIFWG operator which has the associated weighting vector $\omega=\left(\omega_{1}, \omega_{2}, \ldots, \omega_{n}\right)^{T}$ and aggregate the given decision matrix $\widetilde{R}=\left(\widetilde{r}_{i j}\right)_{m \times n}$ into group integrated attribute values $\widetilde{r}_{i}$ :

$$
\begin{aligned}
\tilde{r}_{i} & =\operatorname{TzFIFWA}_{\omega}\left(\tilde{a}_{1}, \tilde{a}_{2}, \ldots, \tilde{a}_{n}\right) \\
& =\sum_{j=1}^{n} \omega_{j} \tilde{a}_{j}=\left(1-\prod_{j=1}^{n}\left(1-\mu_{j}\right)^{\omega_{j}}, \prod_{j=1}^{n}\left(\gamma_{j}\right)^{\omega_{j}}\right) \\
& =\left(\left[1-\prod_{j=1}^{n}\left(1-\mu_{1 j}\right)^{\omega_{j}}, 1-\prod_{j=1}^{n}\left(1-\mu_{2 j}\right)^{\omega_{j}},\right.\right.
\end{aligned}
$$




$$
\begin{aligned}
& \left.1-\prod_{j=1}^{n}\left(1-\mu_{3 j}\right)^{\omega_{j}}, 1-\prod_{j=1}^{n}\left(1-\mu_{4 j}\right)^{\omega_{j}}\right], \\
& \left.\left[\prod_{j=1}^{n}\left(\gamma_{1 j}\right)^{\omega_{j}}, \prod_{j=1}^{n}\left(\gamma_{2 j}\right)^{\omega_{j}}, \prod_{j=1}^{n}\left(\gamma_{3 j}\right)^{\omega_{j}}, \prod_{j=1}^{n}\left(\gamma_{4 j}\right)^{\omega_{j}}\right]\right)
\end{aligned}
$$

or

$$
\begin{aligned}
\widetilde{r}_{i}= & \operatorname{TzFIFWG}_{\omega}\left(\widetilde{a}_{1}, \tilde{a}_{2}, \ldots, \tilde{a}_{n}\right) \\
= & \sum_{j=1}^{n} \omega_{j} \widetilde{a}_{j}=\left(\prod_{j=1}^{n}\left(\mu_{j}\right)^{\omega_{j}}, 1-\prod_{j=1}^{n}\left(1-\gamma_{j}\right)^{\omega_{j}}\right) \\
= & {\left[\prod_{j=1}^{n}\left(\mu_{1 j}\right)^{\omega_{j}}, \prod_{j=1}^{n}\left(\mu_{2 j}\right)^{\omega_{j}},\right.} \\
& \left.\prod_{j=1}^{n}\left(\mu_{3 j}\right)^{\omega_{j}}, \prod_{j=1}^{n}\left(\mu_{4 j}\right)^{\omega_{j}}\right], \\
& {\left[\begin{array}{l}
1-\prod_{j=1}^{n}\left(1-\gamma_{1 j}\right)^{\omega_{j}}, 1-\prod_{j=1}^{n}\left(1-\gamma_{2 j}\right)^{\omega_{j}}, \\
\left.\left.1-\prod_{j=1}^{n}\left(1-\gamma_{3 j}\right)^{\omega_{j}}, 1-\prod_{j=1}^{n}\left(1-\gamma_{4 j}\right)^{\omega_{j}}\right]\right) .
\end{array}\right.}
\end{aligned}
$$

Step 2. Utilize the correlation coefficient (8) to (11) of TzFIFNs to derive the closeness between the overall group integrated attribute values $\widetilde{r}_{i}$ and the TzFIFN positive ideal value $\widetilde{r}^{+}$, where $\widetilde{r}^{+}=\langle[1,1,1,1],[0,0,0,0]\rangle$.

Step 3. Rank alternatives $A_{i}, i=1,2, \ldots, m$, and select the best in accordance with the highest closeness obtained from Step 2.

\section{Numerical Illustration}

A company intends to select one person to take the position of Assistant Manager from four candidates. Five indicators (attributes) must be evaluated. They are shown as follows:

(i) technical skill $\left(G_{1}\right)$,

(ii) professional ability $\left(G_{2}\right)$,

(iii) creative ability $\left(G_{3}\right)$,

(iv) analytical skill $\left(G_{4}\right)$,

(v) leadership ability $\left(G_{5}\right)$.

The weights of the indicators are $=\left(\omega_{1}, \omega_{2}, \ldots, \omega_{n}\right)^{T}=$ $(0.18,0.26,0.15,0.19,0.22)^{T}$. The individual attributes of each candidate are to be evaluated in order to come to a good decision. The decision matrix $\widetilde{R}=\left(\widetilde{r}_{i j}\right)_{m \times n}$ is given as follows:

$\widetilde{R}=\left(\begin{array}{c}G_{1} \\ \langle[0.15,0.21,0.32,0.42],[0.19,0.23,0.42,0.51]\rangle \\ \langle[0.21,0.33,0.45,0.49],[0.12,0.19,0.23,0.35]\rangle \\ \langle[0.13,0.19,0.31,0.39],[0.11,0.23,0.27,0.31]\rangle \\ \langle[0.18,0.25,0.32,0.43],[0.17,0.23,0.34,0.41]\rangle\end{array}\right.$

$G_{2}$

$\langle[0.11,0.19,0.23,0.31],[0.13,0.24,0.29,0.35]\rangle$

$\langle[0.13,0.15,0.25,0.34],[0.19,0.21,0.26,0.32]\rangle$

$\langle[0.21,0.23,0.29,0.42],[0.28,0.34,0.41,0.46]\rangle$

$\langle[0.22,0.25,0.39,0.43],[0.33,0.35,0.41,0.45]\rangle$

$G_{3}$

$\langle[0.17,0.27,0.37,0.43],[0.11,0.19,0.23,0.30]\rangle$

$\langle[0.21,0.33,0.42,0.51],[0.25,0.32,0.41,0.45]\rangle$

$\langle[0.15,0.20,0.35,0.49],[0.22,0.26,0.35,0.42]\rangle$

$\langle[0.16,0.23,0.36,0.39],[0.27,0.35,0.41,0.46]\rangle$

$G_{4}$

$\langle[0.25,0.35,0.45,0.55],[0.15,0.20,0.32,0.41]\rangle$

$\langle[0.21,0.32,0.43,0.56],[0.12,0.23,0.33,0.39]\rangle$

$\langle[0.13,0.15,0.25,0.29],[0.11,0.25,0.34,0.41]\rangle$

$\langle[0.15,0.23,0.41,0.44],[0.21,0.32,0.39,0.46]\rangle$

$G_{5}$

$\langle[0.15,0.23,0.34,0.47],[0.25,0.28,0.34,0.45]\rangle$

$\langle[0.18,0.25,0.36,0.43],[0.26,0.37,0.47,0.51]\rangle$

$\langle[0.19,0.23,0.35,0.43],[0.27,0.31,0.41,0.52]\rangle\rangle$.

$\langle[0.24,0.34,0.45,0.61],[0.15,0.25,0.28,0.30]\rangle)$

Step 1. Utilize the decision information given in the matrix $\widetilde{R}$, the TzFIFWA operator, and the weighting vector of the attributes $\omega=\left(\omega_{1}, \omega_{2}, \ldots, \omega_{n}\right)^{T}=(0.18,0.26,0.15,0.19$, $0.22)^{T}$ and aggregate the decision matrix $\widetilde{R}$. The collective values are as follows:

$$
\begin{array}{r}
\tilde{r}_{1}=\langle[0.1629,0.2470,0.3375,0.4346], \\
[0.1610,0.2297,0.3159,0.3986]\rangle, \\
\tilde{r}_{2}=\langle[0.1832,0.2673,0.3744,0.4598], \\
[0.1789,0.2532,0.3245,0.3937]\rangle, \\
\tilde{r}_{3}=\langle[0.1677,0.2036,0.3092,0.4057], \\
[0.1896,0.2813,0.3584,0.4248]\rangle, \\
\tilde{r}_{4}=\langle[0.1956,0.2642,0.3914,0.4720], \\
[0.2192,0.2963,0.3729,0.4078]\rangle .
\end{array}
$$

Step 2. Utilize the correlation coefficient of TzFIFNs to derive the closeness between the overall group integrated attribute values $\tilde{r}_{i}$ and the TzFIFN positive ideal value $\tilde{r}^{+}$, where 
$\widetilde{r}^{+}=\langle[1,1,1,1],[0,0,0,0]\rangle$. Using (8) to (11), the correlation coefficients are calculated using the following formula:

$$
\begin{gathered}
K_{\mathrm{TzFIFS}}(A, B)=\frac{C_{\mathrm{TzFIFS}}(A, B)}{\sqrt{E_{\mathrm{TzFIFS}}(A) \cdot E_{\mathrm{TzFIFS}}(B)}}, \\
0 \leq K_{\mathrm{TzFIFS}}(A, B) \leq 1 .
\end{gathered}
$$

Hence the calculated values are given as follows:

$$
\begin{array}{ll}
C_{\mathrm{TzFIFS}}\left(\widetilde{r}_{1}, \widetilde{r}^{+}\right)=0.2955, & K_{\mathrm{TzFIFS}}\left(\widetilde{r}_{1}, \widetilde{r}^{+}\right)=0.4663, \\
C_{\mathrm{TzFIFS}}\left(\widetilde{r}_{2}, \widetilde{r}^{+}\right)=0.3212, & K_{\mathrm{TzFIFS}}\left(\widetilde{r}_{2}, \widetilde{r}^{+}\right)=0.5140, \\
C_{\mathrm{TzFIFS}}\left(\widetilde{r}_{3}, \widetilde{r}^{+}\right)=0.2715, & K_{\mathrm{TzFIFS}}\left(\widetilde{r}_{3}, \widetilde{r}^{+}\right)=0.4332, \\
C_{\mathrm{TzFIFS}}\left(\widetilde{r}_{4}, \widetilde{r}^{+}\right)=0.3308, & K_{\mathrm{TzFIFS}}\left(\widetilde{r}_{4}, \widetilde{r}^{+}\right)=0.5347 .
\end{array}
$$

Step 3. Rank alternatives $A_{i}, i=1,2, \ldots, m$, from the highest closeness value (correlation coefficient) obtained from Step 2; the result is obtained as follows:

$$
A_{4}>A_{2}>A_{1}>A_{3} .
$$

Hence the best alternative is $A_{4}$.

Following the MADM model using the TzFIFWA operator, we have the following numerical results for the MADM model using TzFIFWG operator.

Step 1. Utilize the decision information given in the matrix $\widetilde{R}_{k}$, the TzFIFWG operator, and the weighting vector of the attributes $\omega=\left(\omega_{1}, \omega_{2}, \ldots, \omega_{n}\right)^{T}=(0.18,0.26,0.15,0.19$, $0.22)^{T}$ and aggregate the same decision matrix $\widetilde{R}$. The collective values are as follows:

$$
\begin{gathered}
\tilde{r}_{1}=\langle[0.1553,0.2388,0.3245,0.4202], \\
[0.1695,0.2325,0.3236,0.4088]\rangle \\
\tilde{r}_{2}=\langle[0.1792,0.2514,0.3608,0.4467], \\
[0.1906,0.2654,0.3431,0.4045]\rangle \\
\tilde{r}_{3}=\langle[0.1635,0.2006,0.3059,0.3973], \\
\quad[0.2094,0.2857,0.3385,0.4347]\rangle \\
\tilde{r}_{4}=\langle[0.1917,0.2600,0.3874,0.4596], \\
[0.2331,0.3024,0.3670,0.4163]\rangle .
\end{gathered}
$$

Step 2. Utilize the correlation coefficient of TzFIFNs to derive the closeness between the overall group integrated attribute values $\tilde{r}_{i}$ and the TzFIFN positive ideal value $\widetilde{r}^{+}$, where $\tilde{r}^{+}=\langle[1,1,1,1],[0,0,0,0]\rangle$. Using (8) to (11), the correlation coefficients are calculated as follows:

$$
\begin{array}{ll}
C_{\mathrm{TzFIFS}}\left(\widetilde{r}_{1}, \widetilde{r}^{+}\right)=0.2847, & K_{\mathrm{TzFIFS}}\left(\widetilde{r}_{1}, \widetilde{r}^{+}\right)=0.4493, \\
C_{\mathrm{TzFIFS}}\left(\widetilde{r}_{2}, \widetilde{r}^{+}\right)=0.3095, & K_{\mathrm{TzFIFS}}\left(\widetilde{r}_{2}, \widetilde{r}^{+}\right)=0.4966, \\
C_{\mathrm{TzFIFS}}\left(\widetilde{r}_{3}, \widetilde{r}^{+}\right)=0.2673, & K_{\mathrm{TzFIFS}}\left(\widetilde{r}_{3}, \widetilde{r}^{+}\right)=0.4281, \\
C_{\mathrm{TzFIFS}}\left(\widetilde{r}_{4}, \widetilde{r}^{+}\right)=0.3246, & K_{\mathrm{TzFIFS}}\left(\widetilde{r}_{4}, \widetilde{r}^{+}\right)=0.5270 .
\end{array}
$$

Step 3. Rank alternatives $A_{i}, i=1,2, \ldots, m$, from the highest closeness (correlation coefficient) obtained from Step 2; the result obtained is

$$
A_{4}>A_{2}>A_{1}>A_{3} .
$$

Hence the best alternative is $A_{4}$.

\section{Conclusion}

In this paper an MADM model was proposed based on the correlation coefficient of TzFIFS for ranking the alternatives together with WA and WG operators. The trapezoidal fuzzy intuitionistic fuzzy weighted averaging (TzFIFWA) operator and the trapezoidal fuzzy intuitionistic fuzzy weighted geometric (TzFIFWG) operator were used to aggregate the trapezoidal fuzzy intuitionistic fuzzy information given in a decision matrix. A numerical illustration was given to show the effectiveness of the proposed approach in using correlation coefficient of TzFIFSs because it preserved the linear relationship between the variables.

\section{Conflict of Interests}

The authors declare that there is no conflict of interests regarding the publication of this paper.

\section{References}

[1] K. T. Atanassov, "Intuitionistic fuzzy sets," Fuzzy Sets and Systems, vol. 20, no. 1, pp. 87-96, 1986.

[2] K. T. Atanassov, "Operators over interval valued intuitionistic fuzzy sets," Fuzzy Sets and Systems, vol. 64, no. 2, pp. 159-174, 1994.

[3] K. Atanassov, Intuitionistic Fuzzy Sets: Theory and Applications, Physica, New York, NY, USA, 1999.

[4] K. Atanassov and G. Gargov, "Interval valued intuitionistic fuzzy sets," Fuzzy Sets and Systems, vol. 31, no. 3, pp. 343-349, 1989.

[5] F. Liu and X. H. Yuan, "Intuitionistic fuzzy sets of fuzzy numbers," Fuzzy Systems and Mathematics, vol. 21, no. 1, pp. 8891, 2007.

[6] E. Szmidt and J. Kacprzyk, "Distances between intuitionistic fuzzy sets," Fuzzy Sets and Systems, vol. 114, no. 3, pp. 505-518, 2000.

[7] E. Szmidt and J. Kacprzyk, "Using intuitionistic fuzzy sets in group decision making," Control and Cybernetics, vol. 31, no. 4, pp. 1037-1053, 2002.

[8] E. Szmidt and J. Kacprzyk, "A consensus-reaching process under intuitionistic fuzzy preference relations," International Journal of Intelligent Systems, vol. 18, no. 7, pp. 837-852, 2003.

[9] P. Burillo, H. Bustince, and V. Mohedano, "Some definitions of intuitionistic fuzzy number," in Proceedings of the 1st Workshop on Fuzzy Based Expert Systems: Fuzzy Bulgarian Enthusiasts, Sofia, Bulgaria, September 1994.

[10] F. E. Boran, S. Genç, M. Kurt, and D. Akay, "A multi-criteria intuitionistic fuzzy group decision making for supplier selection with TOPSIS method," Expert Systems with Applications, vol. 36, no. 8, pp. 11363-11368, 2009. 
[11] D. F. Chen, L. Zhang, and J. Jiao, “Triangle fuzzy number intuitionistic fuzzy aggregation operators and their application to group decision making," in Artificial Intelligence and Computational Intelligence: Proceedings of the International Conference, AICI 2010, Sanya, China, October 23-24, 2010, Part II, vol. 6320 of Lecture Notes in Computer Science, pp. 350-357, Springer, Berlin, Germany, 2010.

[12] G. Wei, "Some induced geometric aggregation operators with intuitionistic fuzzy information and their application to group decision making," Applied Soft Computing Journal, vol. 10, no. 2, pp. 423-431, 2010.

[13] X. F. Wang, "Fuzzy number intuitionistic fuzzy geometric aggregation operators and their application to decision making," Control and Decision, vol. 23, no. 6, pp. 607-612, 2008.

[14] X. Zhang and P. D. Liu, "Method for aggregating triangular fuzzy intuitionistic fuzzy information and its application to decision making," Technological and Economic Development of Economy, vol. 16, no. 2, pp. 280-290, 2010.

[15] Z.S. Xu and R. R. Yager, "Some geometric aggregation operators based on intuitionistic fuzzy sets," International Journal of General Systems, vol. 35, no. 4, pp. 417-433, 2006.

[16] Z. S. Xu and J. Chen, "An approach to group decision making based on interval-valued intuitionistic judgment matrices," System Engineering Theory and Practice, vol. 27, no. 4, pp. 126133, 2007.

[17] Z. S. Xu and J. Chen, "On geometric aggregation over intervalvalued intuitionistic fuzzy information," in Proceedings of the 4th International Conference on Fuzzy Systems and Knowledge Discovery (FSKD '07), vol. 2, pp. 466-471, August 2007.

[18] F. Herrera, L. Martinez, and P. J. Sanchez, "Managing nonhomogenous information in group decision making," European Journal of Operational Research, vol. 116, pp. 115-132, 1999.

[19] D. F. Li, "Multiattribute decision making models and methods using intuitionistic fuzzy sets," Journal of Computer and System Sciences, vol. 70, no. 1, pp. 73-85, 2005.

[20] D. F. Li and J. X. Nan, "Extension of TOPSIS for multi-attribute group decision making under Atanassov IFS environments," International Journal of Fuzzy System Applications, vol. 1, no. 4, pp. 47-61, 2011.

[21] D. J. Power, Engineering Effective Decision Support Technologies: New Models and Applications, IGI Global, Hershey, Pa, USA, 2013.

[22] D. G. Park, Y. C. Kwun, J. H. Park, and I. Y. Park, "Correlation coefficient of interval-valued intuitionistic fuzzy sets and its application to multiple attribute group decision making problems," Mathematical and Computer Modelling, vol. 50, no. 9-10, pp. 1279-1293, 2009.

[23] J. P. Robinson and E. C. H. Amirtharaj, "A short primer on the correlation coefficient of vague sets," International Journal of Fuzzy System Applications, vol. 1, no. 2, pp. 55-69, 2011.

[24] J. P. Robinson and E. C. H. Amirtharaj, "Extended TOPSIS with correlation coefficient of Triangular Intuitionistic fuzzy sets for Multiple Attribute Group Decision Making," International Journal of Decision Support System Technology, vol. 3, no. 3, pp. 15-40, 2011.

[25] J. P. Robinson and E. C. H. Amirtharaj, "Vague correlation coefficient of interval Vague sets," International Journal of Fuzzy System Applications, vol. 2, no. 1, pp. 18-34, 2012.

[26] J. P. Robinson and E. C. H. Amirtharaj, "A aearch for the correlation coefficient of triangular and trapezoidal intuitionistic fuzzy sets for multiple attribute group decision making," in
Communications in Computer and Information Science, vol. 283, pp. 333-342, Springer, Berlin, Germany, 2012. 


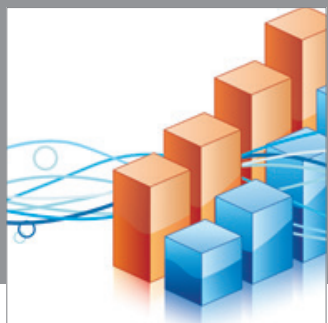

Advances in

Operations Research

mansans

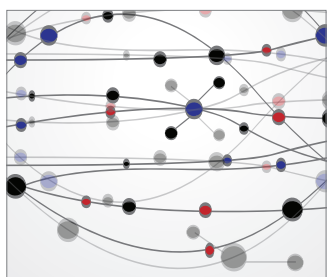

The Scientific World Journal
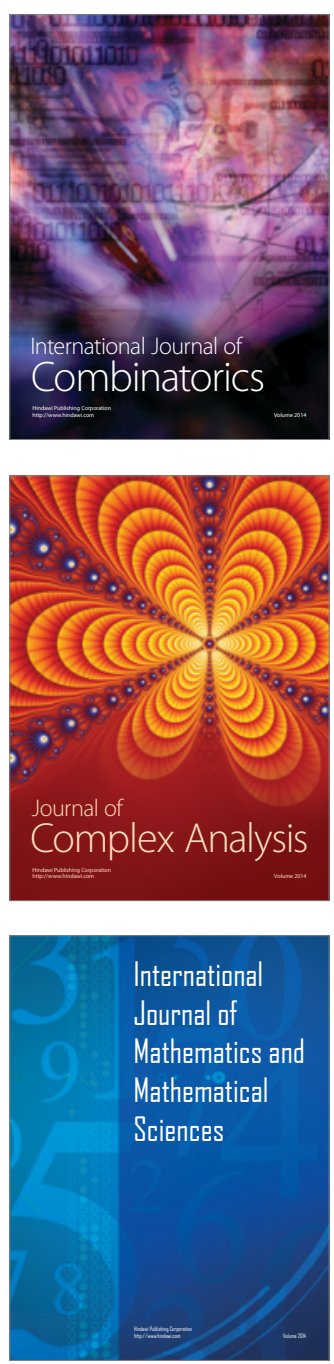
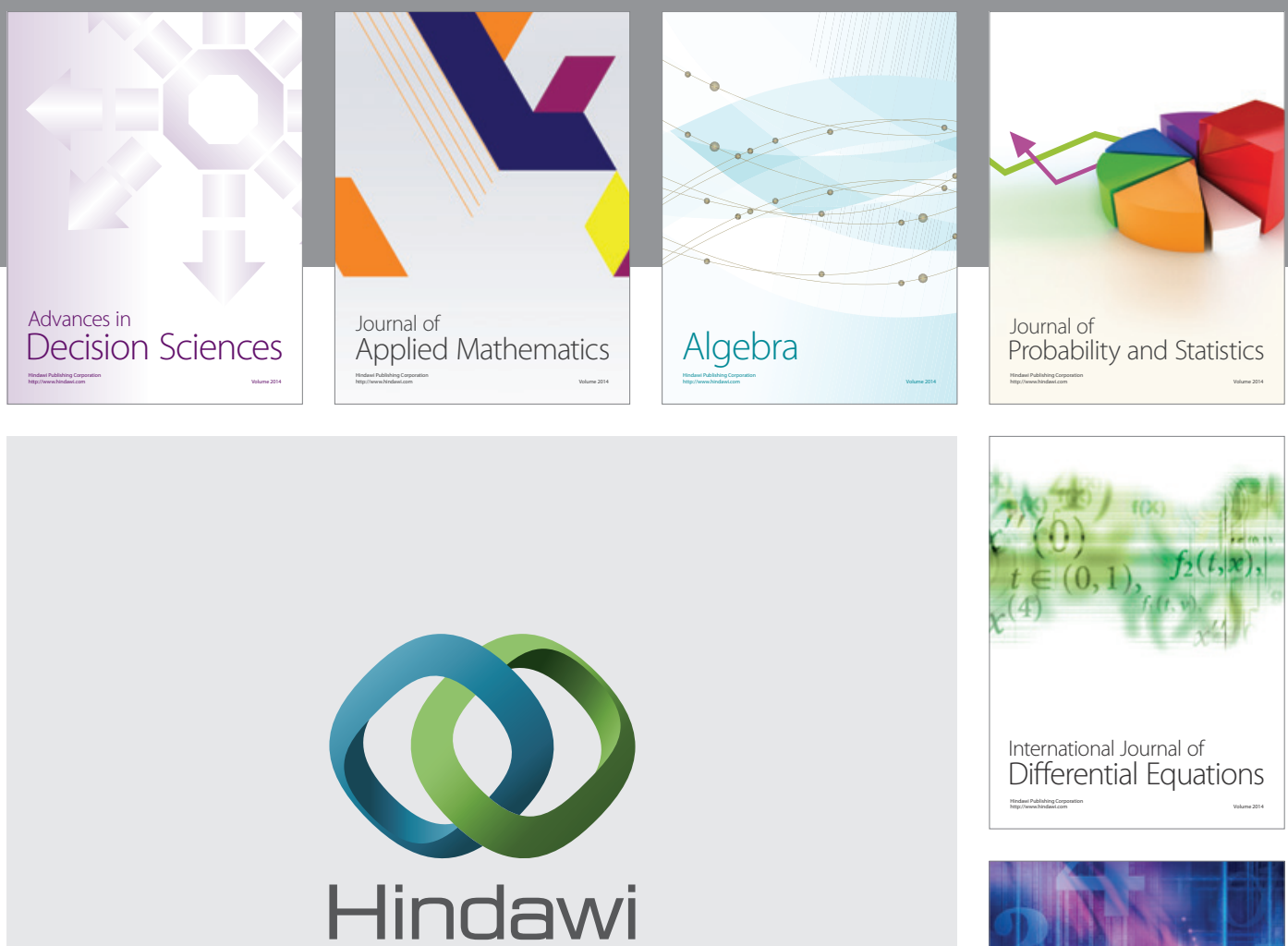

Submit your manuscripts at http://www.hindawi.com
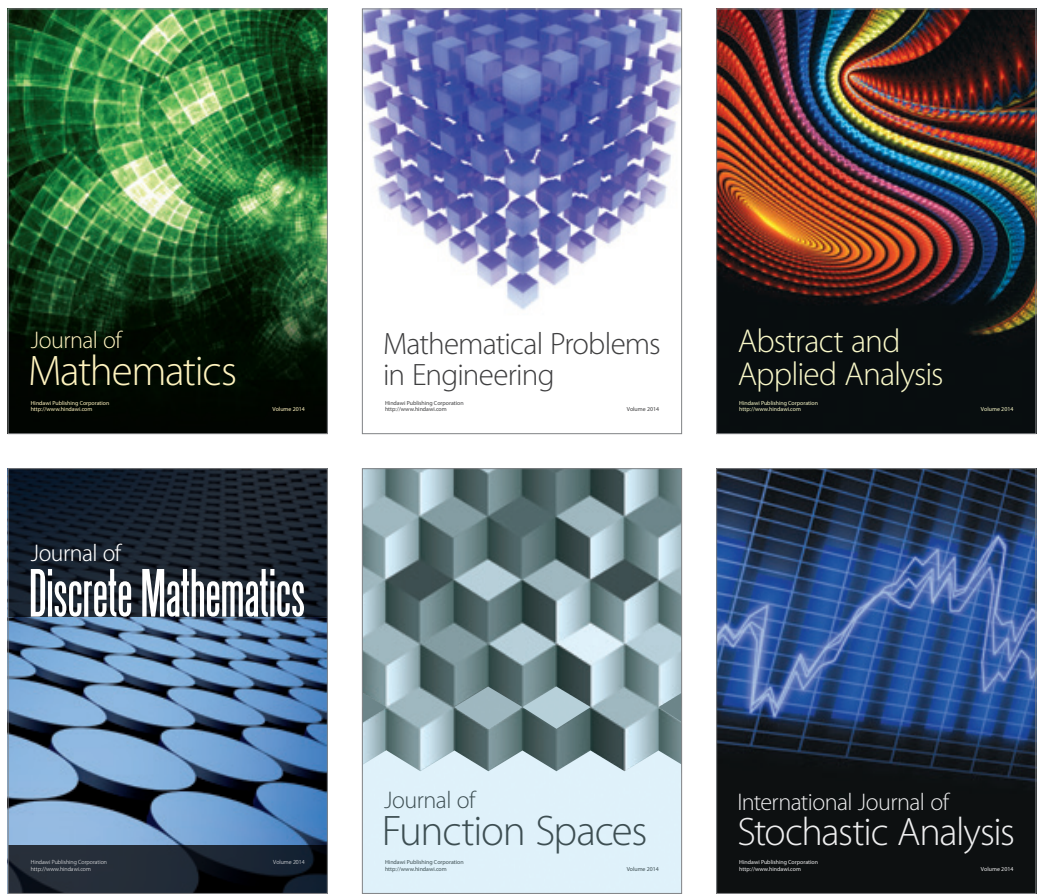

Journal of

Function Spaces

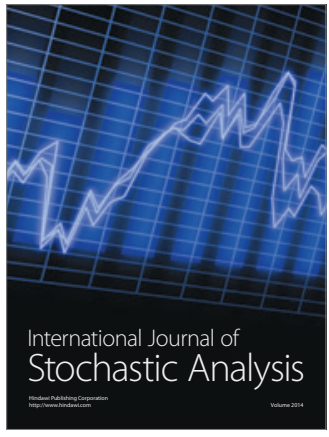

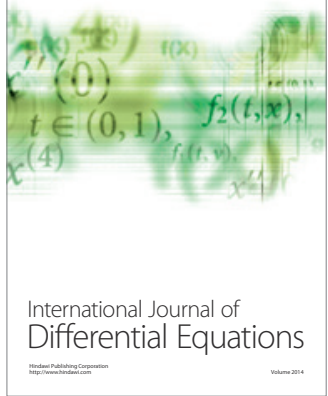
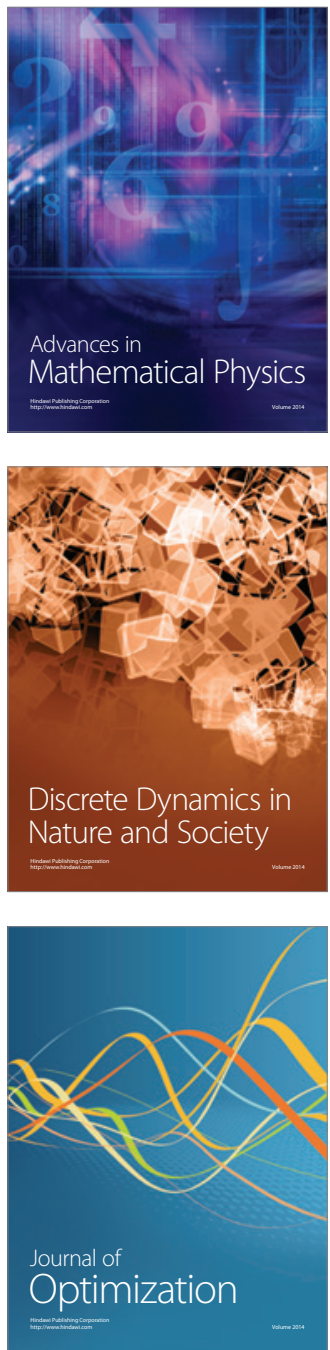